\title{
Curvature as a collective coordinate in enhanced sampling membrane simulations
}

\author{
Benjamin Bouvier* \\ Laboratoire de Glycochimie, des Antimicrobiens et des Agroressources, CNRS \\ UMR7378/Université de Picardie Jules Verne, 10, rue Baudelocque, 80039 Amiens Cedex, \\ France. \\ E-mail: benjamin.bouvier@u-picardie.fr
}

\section{Abstract}

The plasticity of membranes plays an important functional role in cells, cell components and micelles, where bending, budding and remodeling implement numerous recognition and communication processes. Comparatively, molecular simulation methods to induce, control and quantitatively characterize such deformations remain scarce. This work defines a novel collective coordinate associated with membrane bending, which strives to combine realism (by preserving the notion of local atomic curvatures) and low computational cost (allowing its evaluation at every time step of a molecular dynamics simulation). Enhanced sampling simulations along this conformational coordinate provide convenient access to the underlying bending free energy landscape. To showcase its potential, the method is applied to three state-of-the-art problems: the determination of the bending free energy landscape of a POPE bilayer, the formation of a POPE liposome, and the study of the influence of the Pseudomonas quinolone signal on the budding of Gram-negative bacterial outer membranes.

\section{Introduction}

Membranes, the ubiquitous delimiters of cells, cell components and micelles, have long been reduced to a passive structural role. However, over the years, substantial evidence that their ability to deform under the influence of diverse factors can be instrumental to biological function has accumulated. Local membrane curvature induced by nanoscale topography is known to act as a biochemical signal, modulating cell signaling ${ }^{1}$ and allowing cells to sense and adapt to their environment. ${ }^{2}$ In bacteria, the budding of membranes initiates the formation of payload-containing vesicles, employed as a means of communication between cells which cleverly combines the fast diffusion of small molecules and the protection offered by encapsulation. ${ }^{3}$ The fluidity of bacterial membranes can also be a crucial factor in the indirect recognition of their targets by antibacterial peptides. ${ }^{4}$ Finally, membrane remodeling plays a role in host-pathogen interactions via the trapping and transport of host and parasite components. ${ }^{5}$

Zooming in at intracellular scales reveals that membrane curvature also plays an important part in the functions of several organelles. In the endoplasmic reticulum, nascent proteins are synthetized and folded inside cisternae delimited by highly curved membrane components, then sorted and transported in membrane vesicles of diverse shapes and sizes. ${ }^{6} \mathrm{Mi}-$ tochondria feature an extensively folded inner membrane, whose invaginations (called cristae) adopt specific shapes, sizes and densities depending upon the current energetic requirements of the cell, optimizing the effectiveness of the transmembrane proton gradient to max- 
imize ATP production. ${ }^{7}$ Understanding vesiculation processes, based on the nature and plasticity of the membranes, is also essential for the design of synthetic vesicles for drug design and delivery. ${ }^{8}$

Membrane curvature is generally modulated by clusters of proteins with specific shapes, acting as scaffolds to bend the membrane into tubules, vesicles or other structures. ${ }^{9}$ However, the protein binding event does not necessarily come first: in many cases, at low surface densities, proteins enter a recognition and sensing mode in which they detect pre-existing curvatures of membrane components; if this recognition is succesful, the scaffolding mode, which occurs at higher protein surface densities, is triggered. ${ }^{10}$ Studying the mechanisms and energetics of membrane deformation is thus essential even in the absence of scaffolding proteins.

Molecular simulations are a precious tool for the study of the deformability of membranes. With the help of coarse-grained models, micrometer- and/or microsecond-scale simulations are now nearing feasability. ${ }^{11,12}$ However, very slow or energetically unfavorable processes remain beyond reach of even such simplified models, especially when estimating free energies differences for which exhaustive sampling is required. Consequently, biased simulations in which the conformational space of a membrane system is modified along a given collective coordinate to enhance sampling are still very relevant today. ${ }^{13,14}$ The effectiveness of such methods depends crucially on how well the collective coordinate is able to map the relevant span of conformational space. The majority of coordinates used to describe the deformation of membrane systems are based on the Helfrich model $^{15}$ in which the free energy of a tensionfree membrane patch is expressed as:

$$
F_{\text {bend }}=\int_{A}\left(\frac{\kappa}{2}\left(C_{1}+C_{2}-C_{0}\right)^{2}+\bar{\kappa} K_{G}\right) d A
$$

which involves the local principal curvatures $C_{1}$ and $C_{2}$ and Gaussian curvature $K_{G}=C_{1} C_{2}$, as well as three parameters: the bending modulus $\kappa$, the Gaussian curvature modulus $\bar{\kappa}$ and the spontaneous curvature $C_{0}$. Unfortunately, the determination of these parameters for a given membrane composition has proven particularly challenging both experimentally and computationally. ${ }^{16,17}$ The determination of local curvatures at the membrane surface atoms stumbles on the non-trivial issue of the realtime reconstruction of a smooth, continuous surface from the instantaneous atomic positions. Fitting the surface using functions such as quadric patches ${ }^{18}$ is time-consuming and very sensitive to thermal noise. Denoising can be addressed using Morse theory: the MorseSmale (MS) complex partitions a mesh or scalar field into regions having uniform gradient flow (i.e., containing lines that originate at a given minimum and terminate at an associated maximum) and can help distinguish salient topological features on the surface from nonsignificant ${ }^{19}$ or nonpersistent ones due to noise. ${ }^{20}$ However, computing an adequate approximation of the MS complex remains an algorithmically complex and computationally costly operation. ${ }^{21}$ Alternately, these theoretical hurdles can be altogether bypassed by designing phenomenological approaches in which membrane curvature is manipulated implicitly via its relationship with other, more straightforward measures. As an example, a recent study uses the approximate local density at the center of a membrane patch, obtained from the distances between the centers of mass of every lipid and that of the patch: acting on this coordinate expels atoms from the membrane plane, inducing out-of-plane bending of the entire membrane. ${ }^{22}$ While effective and simple, such coordinates can only induce a limited repertoire of deformations; in addition, their complex relationship with membrane curvature can complicate the interpretation of quantitative results.

Because of these difficulties, and despite the growing importance of membrane simulations, there is a dearth of implementations of conformational coordinates that simultaneously provide a realistic approximation of curvature and satisfy the stringent computational cost limitations inherent to being repeatedly evaluated at millions of timesteps. This study sets out to design such a coordinate, with the follow- 
ing specifications: (i) preserve the notion of local curvatures, favoring generality; (ii) exploit salient features on the membrane surface and their persistence over time to achieve a better approximation of the curvatures; (iii) provide access from the most popular molecular dynamics packages. The method is described in the following section. Its effectiveness is showcased further down, on typical membrane systems and simulation setups.

\section{Methods}

\subsection{Defining local curvature}

Following Hoppe's seminal work, ${ }^{23}$ the local curvature $\kappa_{i}$ at an atom $i$ at coordinates $\mathbf{X}_{i}$ on the membrane surface can be approximated as:

$$
\kappa_{i}=\frac{\min _{j \in 1, \ldots 3} \lambda_{j}}{\sum_{j=1}^{3} \lambda_{j}}
$$

where $\left\{\lambda_{j}, j \in 1 \ldots 3\right\}$ is the set of eigenvalues of the covariance matrix $\mathbf{A}_{i}$ :

$$
\mathbf{A}_{i}(j, k)=\sum_{l \in \mathcal{U}_{i}}\left(\mathbf{X}_{l}(j)-\overline{\mathbf{X}_{i}(j)}\right)\left(\mathbf{X}_{l}(k)-\overline{\mathbf{X}_{i}(k)}\right)^{\mathrm{T}}
$$

$\mathcal{U}_{i}$ is a well-chosen set of neighboring atoms and $\overline{\mathbf{X}_{i}}$ the centroid thereof. The smallest eigenvalue's corresponding eigenvector is normal to the plane defined by the two other dominant eigenvectors: curvature can thus be seen as the relative weight of the out-of-plane component of the local arrangement of atoms. Implementing this definition thus requires overcoming two major hurdles: (i) the determination of a suitable neighborhood $\mathcal{U}_{i}$ of atom $i$ and (ii) the efficient calculation of the eigenvalues $\lambda_{j}$ and their gradient.

\subsection{Delimiting atomic neighbor- hoods}

In most works based on similar definitions of local curvature, the neighborhood $\mathcal{U}_{i}$ of atom $i$ consists of either a fixed number of atoms nearest to $\mathbf{X}_{i}$, or all atoms within a fixed distance of $\mathbf{X}_{i} \cdot{ }^{24}$ Such choices work well for smooth and flat surfaces but can result in severe inaccuracies for surfaces featuring (i) noise and/or (ii) sharp localized features such as edges or creases. Distinguishing signal from noise in the first scenario requires evaluating the typical feature size of both, which is usually non-trivial; in the second scenario, points on either side of a sharp feature usually belong to regions with distinct shapes and curvatures, such that lumping them into the same neighborhood via a simplistic nearestneighbor approach results in an unrealistic averaging. Several methods have been proposed for the denoising, feature identification and segmentation of surfaces. ${ }^{19-21,25-29}$ Many are based on approximations of Reeb graphs or MS complexes and are both difficult to efficiently implement and computationally too demanding to be performed millions of times during a simulation. Albeit conceptually simpler, approaches that do not resort to Morse theory cannot use the topology of the underlying scalar field as a safeguard and can end up being even more computationally expensive. ${ }^{19}$

As a tradeoff between adequate detection of features and computational efficiency, this paper uses a two-step region-growing approach similar to that of Rabbani and coworkers. ${ }^{26}$ First, the traditional approach in which the neighborhood $\mathcal{U}_{i}$ of an atom $i$ consists of a fixed number $n_{\text {neigh }}$ of neighbors (user-defined, default 100) is employed. The eigenvector $\mathbf{N}_{i}$ associated with the smallest eigenvalue of the covariance matrix thus obtained is normal to a plane which represents a coarse local approximation of the surface at atom $i$. The quality of this approximation, which depends on the planearity of the surface, is evaluated by computing atom $i$ 's plane residual $\rho_{i}$ (its distance from the plane along its normal): $\rho_{i}=\left|\mathbf{N}_{i} \cdot \mathbf{X}_{i}-\overline{\rho_{\mathcal{U}_{i}}}\right|$ with $\overline{\rho_{\mathcal{U}_{i}}}=1 / \operatorname{card}\left(\mathcal{U}_{i}\right) \cdot \sum_{k \in \mathcal{U}_{i}} \mathbf{N}_{i} \cdot \mathbf{X}_{k}$. The following procedure is then iterated:

1. Select the atom with the lowest plane residual not yet affected to a region as the seed point from which a new region is grown.

2. Iterate over the neighbors of the current seed that have not yet been affected to regions. Atoms whose normals make an 
angle lower than $\theta_{\max }$ (user-defined, default $5^{\circ}$ ) with that of the seed are added to the current region. Among these, atoms whose plane residuals are less than a threshold value (defined from a userchosen percentile $P_{\text {seed }}$ of the distribution of residuals at all atoms, default 10\%) are added to the list of seeds from which the current region will be expanded. When all neighbors have been iterated over, the current seed is removed from the list of seeds.

3. Repeat step 2 until the seed list for the current region is empty.

4. Add the current region to the list of regions and return to step 1 .

The update of each atom's neighbor list and the growing of regions is performed at regular, user-defined timestep intervals during the simulation to take into account the relative motion of atoms and the evolution of surface features. It is forced when the variation of an atom's local curvature between successive timesteps is larger than a certain user-defined percentage (default $50 \%$ ), to properly account for rapid local deformations that can occur in simulations under strong bias.

When evaluating the curvature at atom $i$ from its neighborhood $\mathcal{U}_{i}$ (equations 2-3), the contribution of each neighbor atom is weighted according to whether it belongs to the same region as $i$ or to a different one. The matrix elements of the (weighted) covariance matrix $\mathbf{A}_{i}$ are computed from the weighted centroids $\overline{\mathbf{X}_{i}}$ :

$$
\mathbf{A}_{i}(j, k)=\sum_{l \in \mathcal{U}_{i}} w_{l}\left(\mathbf{X}_{l}(j)-\overline{\mathbf{X}_{i}(j)}\right)\left(X_{l}(k)-\overline{\mathbf{X}_{i}(k)}\right)^{\mathrm{T}}
$$

$$
\overline{\mathbf{X}_{i}}=\frac{1}{\sum_{l \in \mathcal{U}_{i}} w_{l}} \cdot \sum_{l \in \mathcal{U}_{i}} w_{l} \mathbf{X}_{\mathbf{l}}
$$

with:

$w_{l}=\left\{\begin{array}{ll}W & \text { if } l \text { and } i \text { belong to the same region; } \\ 1 & \text { otherwise }\end{array}\right\}$
$W \gg 1$ is a user-chosen parameter (default 20 ), representing the relative influence on the local curvature of points in the same region compared to points in different regions. Sharply bent areas, in which the direction of the normal vector varies sizeably between nearby atoms, can become fragmented into many regions comprising only a few atoms each. This can contaminate the determination of local curvatures with noise due to insufficient averaging, and raise continuity problems due to the high ratio of atoms that can switch between nearby regions from one frame to the next. Using a nonzero (albeit small) relative weight for points in distinct regions is a tradeoff between alleviating these issues and retaining the aforementioned benefits of using regions.

\subsection{Efficiently computing eigen- values and their gradient}

Computing local membrane curvatures and deriving associated biasing forces at each timestep implies the cost-efficient computation of the eigenvalues of covariance matrix $\mathbf{A}_{i}=\left(\begin{array}{lll}a 11 & a 12 & a 13 \\ a 12 & a 22 & a 13 \\ a 13 & a 23 & a 33\end{array}\right)$ (equation 3) and their derivatives with respect to the atomic coordinates (or gradient). This work exploits the matrix's dimensionality and real symmetric nature to provide a closed-form expression of the eigenvalues, computationally much more efficient than generic matrix diagonlization methods.

The eigenvalues $\lambda_{l}$ of $\mathbf{A}_{i}$ are given by the characteristic equation:

$$
P(\lambda)=\left|\mathbf{A}_{i}-\lambda \mathbf{I}\right|=\lambda^{3}+\gamma \lambda^{2}+\beta \lambda+\alpha=0
$$

with:

$\gamma=-a_{11}-a_{22}-a_{33}$
$\beta=a_{11} a_{22}+a_{11} a_{33}+a_{22} a_{33}-a_{12}^{2}-a_{13}^{2}-a_{23}^{2}$

$\alpha=a_{11} a_{23}^{2}+a_{22} a_{13}^{2}+a_{33} a_{12}^{2}-a_{11} a_{22} a_{33}-2 a_{13} a_{12} a_{23}$

Cardano's method ${ }^{30,31}$ is employed to solve this third-degree equation, yielding the following analytical expression for the eigenvalues (demonstrated in Supporting Information): 


$$
\lambda_{l}=\frac{\sqrt{p}}{3} \xi_{l}-\frac{1}{3} \gamma
$$

with:

$$
\begin{aligned}
\xi_{1} & =2 \cos \phi \\
\xi_{2} & =-\cos \phi-\sqrt{3} \sin \phi \\
\xi_{3} & =-\cos \phi+\sqrt{3} \sin \phi \\
\phi & =\frac{1}{3} \arctan \frac{\sqrt{27\left(\alpha\left(q+\frac{27}{4} \alpha\right)+\frac{\beta^{2}}{4}(p-\beta)\right)}}{q} \\
p & =\gamma^{2}-3 \beta \\
q & =-\frac{27}{2} \alpha-\gamma^{3}+\frac{9}{2} \beta \gamma
\end{aligned}
$$

Considering the intricacy of the relationship between the eigenvalues of $\mathbf{A}_{i}$ and the atomic coordinates, the analytical expressions of the partial derivatives $\frac{\partial \lambda_{l}}{\partial \mathbf{X}_{j}(k)}$ required for the calculation of biasing forces were obtained by recursively applying the chain rule to the expression of $\lambda_{l}$ using the Tapenade automatic differentiation engine. ${ }^{32}$

One of the benefits of using Cardano's method is the ability to obtain the eigenvalues without the need to compute the corresponding eigenvectors, which aren't used in the definition of local curvature (equation 2). However, the region-growing algorithm described above requires surface normals, approximated by the eigenvector associated with the smallest eigenvalue of the neighborhood atoms covariance matrix. Thus, computing the eigenvectors does become necessary at regular intervals.

In most cases, the eigenvectors $\mathbf{V}_{l}$ can be obtained very easily from the eigenvalues $\lambda_{l}$, the first two columns $\mathbf{A}_{i}(1)$ and $\mathbf{A}_{i}(2)$ of the covariance matrix and the first two basis vectors $\mathbf{u}_{x}$ and $\mathbf{u}_{y}$ (see Supporting Information for details):

$$
\begin{aligned}
\mathbf{V}_{1} & =\left(\mathbf{A}_{i}(1)-\lambda_{1} \mathbf{u}_{x}\right) \times\left(\mathbf{A}_{i}(2)-\lambda_{1} \mathbf{u}_{y}\right) \\
\mathbf{V}_{2} & =\left(\mathbf{A}_{i}(1)-\lambda_{2} \mathbf{u}_{x}\right) \times\left(\mathbf{A}_{i}(2)-\lambda_{2} \mathbf{u}_{y}\right) \\
\mathbf{V}_{3} & =\mathbf{V}_{1} \times \mathbf{V}_{2}
\end{aligned}
$$

If $\mathbf{V}_{1}$ and $\mathbf{V}_{2}$ are collinear, or if any $\mathbf{A}_{i}(k)-$ $\lambda_{j} \mathbf{u}_{k}$ has a very small norm due to error cancellations, this approach cannot be used and the much slower, but more robust, QL algorithm is employed instead. However, this was never seen to happen in the application examples presented in this paper.

\subsection{Registering membrane con- formations}

The individual components of a membrane (lipid molecules) are known, over sufficiently long timescales, to migrate with respect to one another. ${ }^{33}$ As such, when comparing two conformations of a lipid membrane patch, it makes more sense to compare local curvatures at points that are similarly placed on both surfaces (regardless of the serial numbers of the lipid molecules at these points in each conformation), than to compare curvatures at a specific lipid molecule which might be found in regions of very different topologies even in perfectly superimposable conformations. A mapping of similarly-located atoms between conformations, allowing to track salient features and their evolution over time, is thus required.

This study employs the iterative closest point (ICP) 3D shape-registration method. ICP recursively applies the following procedure. Given a mapping $\mathcal{M}$ between the indices of atoms in the first and second conformation, the goal is to find the optimal rotation $\mathcal{R}$ and translation $\mathcal{T}$ that, once applied to the atoms of the first conformation, minimize the distance between all pairs of points $i$ and $\mathcal{M}(i)$. The $N$ atoms of the two conformations, of respective coordinates $\mathbf{X}_{i}$ and $\mathbf{Y}_{i}$, are first recentered around their respective centroids $\overline{\mathbf{X}}=$ $\frac{1}{N} \sum_{i=1}^{N} \mathbf{X}_{i}$ and $\overline{\mathbf{Y}}=\frac{1}{N} \sum_{i=1}^{N} \mathbf{Y}_{i} . \mathcal{R}$ is obtained by SVD decomposition of the following covariance matrix $\mathbf{A}$ :

$$
\begin{aligned}
\mathbf{A} & =\sum_{i=1}^{N}\left(\mathbf{X}_{i}-\overline{\mathbf{X}}\right) \cdot\left(\mathbf{Y}_{\mathcal{M}(i)}-\overline{\mathbf{Y}}\right)^{\mathrm{T}} \\
\mathbf{U}, \mathbf{S}, \mathbf{V} & =\operatorname{SVD}(\mathbf{A}) \text { such that } \mathbf{A}=\mathbf{U S V}^{\mathrm{T}} \\
\mathcal{R} & =\mathbf{V U}^{\mathrm{T}}
\end{aligned}
$$


In cases where the determinant of $\mathcal{R}$ is negative $(-1)$, the actual rotation matrix is obtained by replacing the third column vector of $\mathcal{R}$ by its opposite. The translation is then obtained as:

$$
\mathcal{T}=\overline{\mathbf{Y}}-\mathcal{R} \cdot \overline{\mathbf{X}}
$$

At the start of the process (iteration 0), atom $i$ in conformation 1 is mapped to atom $i$ in conformation 2: $\mathcal{M}_{0}(i)=i$. At iteration 1 , the transformations $\mathcal{R}_{1}$ and $\mathcal{T}_{1}$ are obtained as previously described; they are used to transform $\mathbf{X}_{i}$ into $\mathbf{X}^{\mathbf{1}}{ }_{i}$. The nearest neighbor $\mathbf{Y}_{j}$ of each $\mathbf{X}^{\mathbf{1}_{i}}$ is then located, defining the new mapping $\mathcal{M}_{1}(i)=j$. The error, defined as the distance between neighbors $d_{1}=\sum_{i=1}^{N}\left\|\mathbf{X}^{\mathbf{1}}{ }_{i}-\mathbf{Y}_{\mathcal{M}_{1}(i)}\right\|$, is also computed. The process is iterated until the distance $d_{\text {final }}$ does not diminish significantly from one iteration to the next. The final mapping $\mathcal{M}_{\text {final }}$ between the transformed coordinates $\mathbf{X}^{\mathrm{final}}{ }_{i}$ and $\mathbf{Y}_{i}$ is used to compare the local curvatures of both structures.

To ensure that the mapping between atoms is as continuous as possible, limiting induced discontinuities in the value of the collective coordinate or its gradient, the ICP is performed at regular intervals of $\Delta t_{\text {map }}$ during the simulation. $\mathcal{M}_{t-\Delta t_{\text {map }}}^{\mathrm{t}}$ is thus obtained by mapping the atoms of frame $t$ onto those of frame $t-\Delta t_{\text {map }}$. The mapping of atoms can then be iteratively propagated to the starting frame of the simulation:

$$
\mathcal{M}_{0}^{t}=\mathcal{M}_{t-\Delta t_{\text {map }}}^{t} \circ \mathcal{M}_{t-2 \Delta t_{\text {map }}}^{t-\Delta t_{\text {map }}} \circ \ldots \circ \mathcal{M}_{0}^{\Delta t_{\text {map }}}
$$

\subsection{Defining the conformational coordinate}

There are multiple ways to construct a scalar collective coordinate describing the overall curvature of a membrane from the scalar field of local atomic curvatures, and selecting the most adequate one is not trivial. This work employs an approach similar to the Euclidean-space root mean square deviation (RMSD) routinely used to compare macromolecular structures. Defining a reference conformation with atomic cur- vatures $\left\{\kappa_{i}^{r e f}, i \in 1 \ldots N\right\}$, the collective coordinate $\Gamma$ at time $t$ is defined from the instantaneous atomic curvatures as:

$$
\Gamma(t)=\sum_{i=1}^{N}\left(\kappa_{\mathcal{M}_{r e f}^{t}(i)}(t)-\kappa_{i}^{r e f}\right)^{2}
$$

$\mathcal{M}_{\text {ref }}^{t}$ is obtained by mapping the atoms at time $t=0$ onto those of the reference structure, then repeatedly propagating the mapping as per equation 13: $\mathcal{M}_{\text {ref }}^{t}=\mathcal{M}_{0}^{t} \circ \mathcal{M}_{\text {ref }}^{0}$. This straightforward definition's main limitation is the fact that, while small values of $\Gamma$ provide a good description of curvature conformational space in the neighborhood of a reference conformation, large values of $\Gamma$ can be simultaneously accomodated by a large number of possibly unrelated structures; the inability to uniformly sample these structures within tractable computational time can raise convergence issues for free energy calculations. However, the same issue affects RMSD and does not prevent it from being used (with the aforementioned caveat in mind) as a conformational coordinate $^{34,35}$ or as a component of more complex collective variables. ${ }^{36,37}$ Furthermore, as in triangulation approaches, the degeneracy between structures sharing similar deviations from a reference can be lifted by simultaneously measuring the deviations from one or more additional references.

\subsection{Implementation details}

The curvature restraint involves numerous neighbor search operations at each timestep, for the calculation of atomic curvatures, the growing of regions and the registration of consecutive structures. To alleviate the corresponding computational effort, a KD-tree algorithm was employed, based on the Nanoflann header-only library $^{38}$ to which a periodic 3D Euclidean metric was added.

The analytical expression of the gradient of $\Gamma$ and its implementation were validated by comparing the computed values to the corresponding numerical derivatives $\delta \Gamma / \delta \mathbf{X}_{i}$ at the limit of small $\delta \mathbf{X}_{i}$ values.

The collective variable was implemented into 
PLUMED $2.5^{39}$ by deriving the Colvar $\mathrm{C}++$ class. This makes it compatible with any of the molecular dynamics engines natively supported by PLUMED. In this work, the GROMACS suite $^{40}$ (versions 2016.4 and 2018.4) was used. The source code of the collective coordinate and the modified Nanoflann headers ( 3000 lines) can be obtained from the author upon request.

\section{Results and discussion}

\subsection{Bending free energy land- scape of a POPE bilayer}

As a proof of concept and to get a better grip on the method's practical use, a simple and common 1-palmitoyl-2-oleoyl-sn-glycero-3phosphoethanolamine (POPE) bilayer patch was used as a 'toy' system; the free energy landscape associated with the bending of this patch was explored using enhanced-sampling MD simulations along the collective coordinate $\Gamma$. The system was described using the DRY MARTINI implicit-solvent coarse-grained formalism, ${ }^{41}$ which has been validated with respect to its explicit-solvent counterpart on the calculation of the bending moduli of common lipids and is thus well suited to the task at hand. A POPE patch placed along the $x y$ plane was constructed, equilibrated and simulated for $750 \mathrm{~ns}$ using well-tempered metadynamics along $\Gamma$ (see Supporting Information for details). The restraint was applied to the head beads on one side of the leaflet (atom set $\mathcal{B}$ of cardinality $N_{\mathcal{B}}$ ). The reference structure for $\mathcal{B}$ was taken as the closest to the average over an unbiased $250 \mathrm{~ns}$ simulation of the system. To favor the convergence of the free energy profile which, as previously mentioned, becomes harder to achieve the further away from the reference the system ventures, the exploration of conformational space was limited using a harmonic 'wall' potential along $\Gamma$, applied for $\Gamma$ values larger than 0.5. Figure 1 shows the free energy profile (FEP) along $\Gamma$ (the convergence of the FEP as a function of simulation time can be checked on Supporting Information figure S1). To visualize the evolution of mem- brane conformations along the bending pathway, the conformations were binned by values of $\Gamma$; the headgroup beads of conformations in each bin were then binned spatially along $x$ and $y$, and the deviation of their $z$ coordinates from $\bar{z}=1 / N_{\mathcal{B}} \sum_{i \in \mathcal{B}} z_{i}$ were computed. The results were then averaged over all conformations in each $\Gamma$ bin and are shown on figure 1 for selected values of $\Gamma$.

Figure 1: Free energy profile and confidence interval for the deformation of a POPE bilayer along $\Gamma$. Insets show the mean relative deviations of the atoms to the average membrane plane along the $z$ axis around selected values of $\Gamma$.

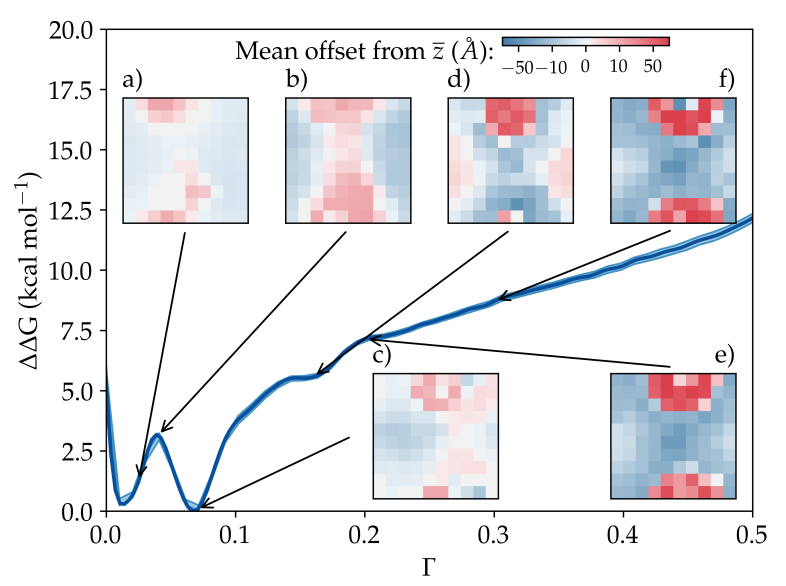

As can be seen, at lower $\Gamma$ values (panels $a-b$ on figure 1) the deformation can be described as a creasing of the membrane along one membrane axis, resulting in a saddle-shaped patch. The evolution of important structural membrane properties along the crease are represented on Supporting Information figures S2S4 and can be summarized as follows. The distribution of areas per lipid is broadened compared to that of a flat patch, both toward lower and higher values; the inner leaflet, more compressed than the outer, is characterized by lower areas. The width of the membrane is also significantly reduced compared to a flat patch. A look at the deuterium order parameters reveals that this is mainly due to the occurence of torsions at the first few carbon atoms of the two POPE acyl chains, during which the chains ro- 
tate away from the membrane normal axis; the statistical orientation of the chain ends, on the other hand, does not differ as much from that of a flat patch. Bent conformations also reveal local interpenetrations of the lipid tails of one leaflet into the other, increasing the separation between lipids within a leaflet and explaining the occurence of the larger area per lipid values mentioned above. Finally, the lateral diffusion of lipids was found to be enhanced upon bending by a factor of 2 to 3 , in line with the local reorganizations observed.

As $\Gamma$ grows larger, rather than continuing along the original crease axis, the bending starts to occur along the axis orthogonal to it (panel c-d); the saddle thus progressively morphs into several distinct peaks and dips (panels e-f). For still larger $\Gamma$ values (not shown on figure 1), the volume of conformational space mapped to a given $\delta \Gamma$ element becomes too large to be exhaustively sampled within typical computational costs; however, a non-exhaustive exploration of the free energy surface reveals that the spatial periodicity of these peaks tends to decrease and their number to increase, forming a very rugged landscape; at this point, the tops of nearby spikes can merge together, eventually splitting the membrane into two components (see Supporting Information figure S5).

Interestingly, the direction of initial bending for the POPE bilayer tends to place the leaflet with the biased headgroups on the inside; in comparable simulations carried out on a DPPC patch, the direction of initial bending appears more arbitrary. This could be due to POPE's strongly negative, compared to DPPC's very weakly positive, spontaneous curvature ${ }^{42}$ and is corroborated by the fact that the distributions of areas per lipids in both leaflets tend to remain more similar in bent patches of DPPC than in POPE (see Supporting Information figure S2).

On figure 2, the correlation between the collective coordinate $\Gamma$ and the number of regions into which the patch is segmented is plain to see but features a substantial fuzzyness: in particular, conformations with $\Gamma \sim 0.25$ have a high probability to comprise anywhere from 7 to 17 distinct regions, and outliers outside this span can also occasionally be found. Indeed, within typical fluctuations around a given structure, spurious movements of individual atoms can cause a region to temporarily split into several near-equivalent ones. Similarly, surface normals tend to vary very quickly in the vicinity of sharp features, which the algorithm can be forced to accomodate by creating several regions consisting of a few (or even a single) atom. This can be seen on the lower panel of figure 2 : the algorithm easily detects the large blue, orange and tan patches which can be considered nearly flat and are assigned their own regions; however, at the hinge between the blue and orange regions, several small regions can be observed which can switch in and out of existence from frame to frame depending on thermal fluctuations. This is not a problem: curvatures and normal vectors at sharp creases on noisy surfaces are ill-defined by definition, and the region-growing algorithm makes sure that the effect on close-lying atoms on flat patches remains minimal.

Interestingly, the saddle-shape conformations observed at the onset of the bending mechanism were also reported in previous studies inducing curvature indirectly (for instance by controlling the lipid density ${ }^{22}$ or along a predefined path). ${ }^{43}$ However, the present study suggests that introducing secondary bending axes is cheaper in free energy than increasing the bend along the axis of the original saddle: the order of magnitude of the free energy penalty is more than twice lower compared to the 80 $\mathrm{kT}$ found by Bubnis et al for the bending of a DOPC patch along a single axis ${ }^{43}$, itself $\sim 5$ times lower than the result obtained by Masone et al on a similar deformation of DPPC. ${ }^{22}$ Compared to methods de facto restricted to the formation of a single feature in the center of the patch, the ability of the method under consideration to gives access to 'rugged' membrane topologies with multiple peaks and throughs is a crucial advantage for the exploration of lowenergy pathways. A larger conformational volume can can also be considered a disadvantage if the $\Gamma$ range it maps to is too small, especially when exhaustive sampling is required; however, such conformational degeneracies can 
Figure 2: Top: distributions of $\Gamma$ values for conformations segmented into a given number of regions by the region-growing algorithm. Central line: median value; box: interquartile range; whiskers: rest of the distribution. Bottom: side view of a POPE bilayer patch with $\Gamma \sim 0.26$ featuring 15 regions; the upper leaflet residues are colored according to the region they belong to, and the heagroup beads (over which curvatures are computed) are represented as spheres.

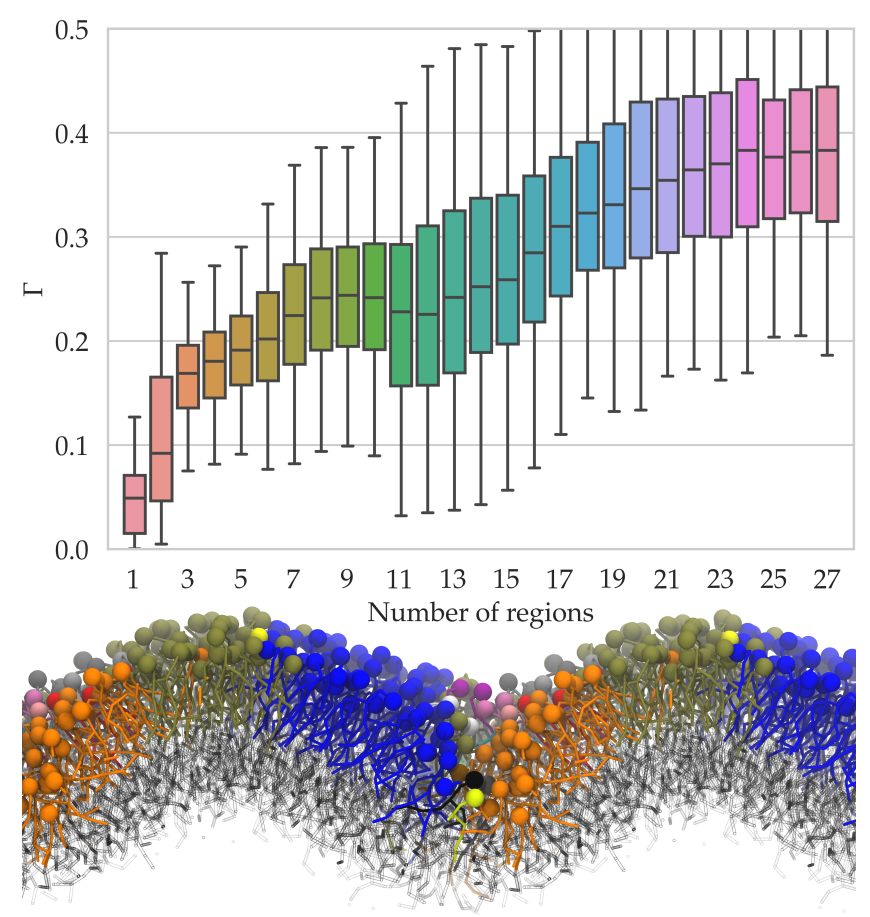

be lifted by measuring each conformation's deviation to several strategically-placed reference structures instead of to a single one. This was applied to the previously described POPE patch, which was subjected to 400 ns of $2 \mathrm{D}$ welltempered metadynamics along the two coordinates $\Gamma_{\text {flat }}$ and $\Gamma_{\text {bent }}$, respectively quantifying the deviation to a flat and bent reference patch (figure 3). The bent reference structure was chosen as representative of conformations with $0.375<\Gamma_{\text {flat }}<0.425$; after minimization, it had a $\Gamma_{\text {flat }}$ value of 0.412 .

The benefits of lifting the degeneracy can clearly be seen: on the $2 \mathrm{D}$ plot, the conformations belonging to both minima along the 1D FES (at $\Gamma_{\text {flat }} \sim 0.02$ and $\Gamma_{\text {flat }} \sim 0.07$, see figure 1) now appear spread out along $\Gamma_{\text {bent }}$ and can thus be distinguished from one another. It
Figure 3: Blue heightmap: 2D free energy surface of the bending of a POPE bilayer with respect to flat ( $\Gamma_{\text {flat }}$, left inset) and bent $\left(\Gamma_{\text {bent }}\right.$, right inset) reference conformations, obtained from $400 \mathrm{~ns}$ of metadynamics along these two coordinates. Black circles: projection on the 2D landscape of the conformations sampled during 750 ns of metadynamics along $\Gamma_{\text {flat }}$ only.

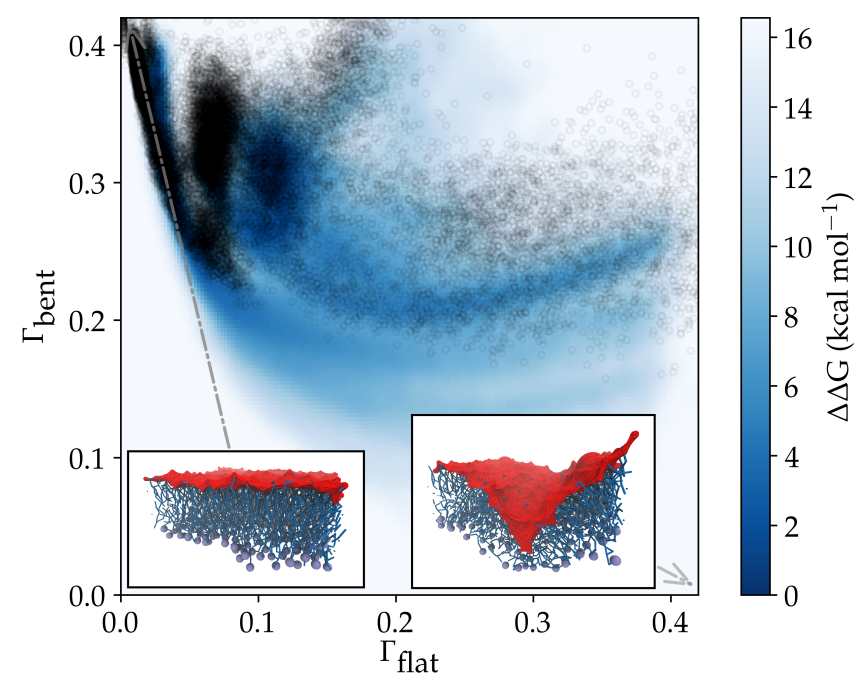

is also clear from the figure that the sampled conformational volume is much more extensive in the 2D metadynamics than in the $1 \mathrm{D}$ simulation, despite the latter's longer duration; indeed, free energy barriers blocking the exploration along a single coordinate can sometimes be easily bypassed by applying bias along additional dimensions. This is especially apparent for conformations with $\Gamma_{\text {flat }}>0.15$, which span a much larger range of $\Gamma_{\text {bent }}$ values in the $2 \mathrm{D}$ simulation compared to the $1 \mathrm{D}$ case. Adding a second curvature coordinate to a metadynamics simulation in PLUMED, however, raises the computational cost by around 65\%. This figure could possibly be reduced by implementing the calculation of distances to several references inside a single PLUMED COLVAR, avoiding redundant calculations of atomic curvatures and housekeeping of large coordinate arrays; in the meantime, the accelerated sampling achieved by the inclusion of an additional coordinate might still be considered worth the added computational cost. 


\subsection{Formation of a POPE lipo- some}

In this scenario, enhanced sampling simulations along the curvature coordinate are used to generate a liposome from a flat membrane patch, as an alternative to $a b$ initio liposome generation methods using mathematical models ${ }^{44}$ or the aggregation of dispersed lipids. This represents a very frequent first step in liposome simulation studies and showcases the breadth of the method's field of applications. Unlike the previous case study, for which the exhaustive sampling of pathways was required, the goal here is to suggest a possible liposome structure as a starting point for further simulations at minimal computational cost; the pathway followed to reach it is of secondary importance and no guarantee is sought that it is the most likely or the lowest in free energy. A cylindrical POPE bilayer patch of approximate diameter $173 \AA$, described using DRY MARTINI, ${ }^{41}$ was constructed and equilibrated. Its structural stability was verified using $250 \mathrm{~ns}$ of unbiased stochastic dynamics simulation. The equilibrated structure was then subjected to 200 ns of well-tempered metadynamics. The set of atoms used for the calculation of local curvatures comprised the headgroups of the lipids on one side of the leaflet pre-equilibration, and their positions in the equilibrated structure were used as reference (see Supporting Information for details).

The main steps of the POPE globule formation mechanism are summarized on figure 4; as explained above, the pathway is one example of many possible outcomes and is only discussed as such. Starting from a flat patch (left panel), the biasing potential progressively builds up until a small lipid subpatch is pushed out of the membrane plane (middle left), initiating membrane bending. The subpatch then grows to become a hinge, around which the membrane progressively folds upon itself. Once the folding is complete (middle right), the system remains conformationally stable while the biasing potential accumulates. The curvature in the hinge regions then increases still further, eventually ejecting 35 lipids from the main patch which then regroup to form a secondary globule. This behavior being undesired, the biased simulation was stopped once the folding of the patch upon itself was complete (which occurs around $170 \mathrm{~ns}$ into the simulation) and the system was allowed to relax via an unbiased simulation. Within $60 \mathrm{~ns}$, the hinge region shifts to equalize both sides of the folded patch and the globule formation completes (right panel on figure 4).

Looking at this mechanism reveals the difficulties of exhaustively sampling the free energy landscape of liposome formation. First, in a nonperiodic patch, different positions for the formation of the hinge become nonequivalent and need to be exhaustively sampled over the entire patch to provide an accurate free energy profile. Second, the folded patch has an overall curvature of $\Gamma>2$ compared to the flat reference structure (see Supporting Information figure $\mathrm{S} 7$ for the graph of $\Gamma$ vs simulation time); further transformations from this state are thus highly degenerate. Other mechanisms, such as the formation of additional hinge points, are probably as likely as the observed ejection phenomenon, and would require the use of additional reference structures to be discriminated. Third, starting the simulation from other representative conformations of the circular patch and/or using slightly different metadynamics parameters can result in a different number of lipids being ejected. This is not specific to POPE: other lipids that are less prone to spontaneous curvature, such as DPPC, exhibit the same behavior. The problem is circumvented here by stopping the biased simulation before the ejection occurs; however, obtaining a complete free energy profile would require the exhaustive sampling of all possible ejection scenarios. All the same, established liposome generation methods such as self-assembly also yield varying distributions of liposome populations and sizes depending on starting conditions. ${ }^{45,46}$. Despite these limitations, this example demonstrates the method's ability for the rapid and easy preparation of liposomes, globules and bicelles, whose formation often span timescales inaccessible to Boltzmann sampling. 
Figure 4: Representative conformations along the formation pathway of a POPE liposome from a circular patch. Approximate simulation times from left to right: 0, 93, 170 and $228 \mathrm{~ns}$.
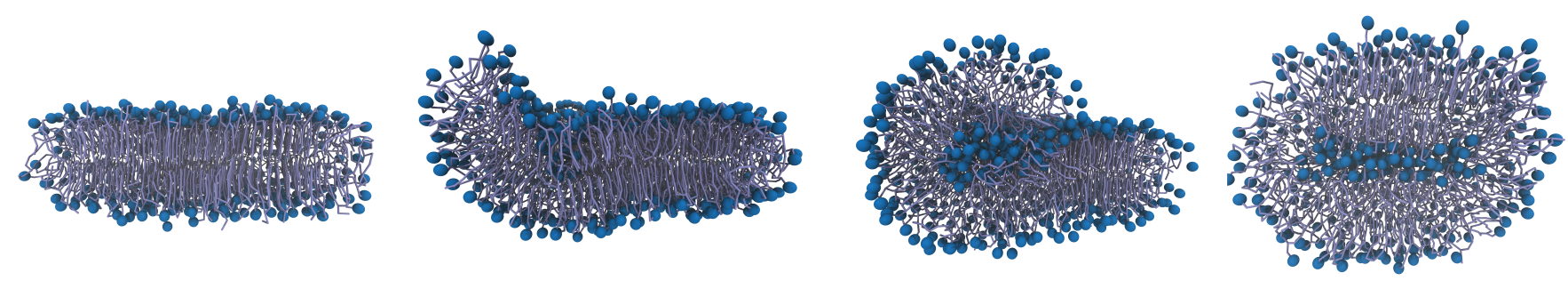

\subsection{Influence of PQS on the bud- ding of the Gram-negative outer membrane}

The Pseudomonas quinolone signal (PQS, 2heptyl-3-hydroxy-4-quinolone) is a quorum sensing ${ }^{47}$ molecule of the Gram-negative bacterium Pseudomonas aeruginosa, a multiresistant pathogen against which the need for new antibiotics has been deemed critical by the World Health Organization. ${ }^{48}$ Among the multiple mechanisms related to quorum sensing, the interaction of PQS with the lipopolysaccharide (LPS) layer of the outer bacterial membrane favors the budding of the latter, eventually forming outer membrane vesicles (OMV) ${ }^{3}$ that encapsulate other molecules (notably, PQS itself, ${ }^{49}$ or siderophores such as pyoverdine ${ }^{50}$ ) and/or are enriched in membrane proteins (notably, FpvA and other TonB-dependent transporters $^{51}$ ). Since OMVs are of paramount importance for bacterial virulence, population control and signalling within the biofilm and hold great promise for targeted drug delivery, ${ }^{52}$ understanding their formation, and the quantitative effect of PQS thereupon, is essential. However, accurate atomic and coarse-grained models of LPS have only recently been made available and the study of large-scale correlated motion in such membranes is still in its infancy. ${ }^{53}$ Here, the curvature restraint is used to study the onset of the budding process and quantify the effect of PQS thereupon.

A MARTINI ${ }^{54}$ model for PQS (figure 5) was created based on, and validated according to, atomistic simulations on this molecule in a solvated environment (see Supporting Information for details). The outer bacterial membrane was represented using a square LPS/POPE bilayer patch of approximate side length $130 \AA$, whose MARTINI parameters were taken from the work of Van Oosten and coworkers ${ }^{55}$ and whose starting structure was obtained by coarsegraining the equilibrated all-atom conformation of Kirschner et al. ${ }^{56}$ After equilibration, the patch was duplicated; one copy was kept pristine while the other was subjected to the insertion of 25 PQS molecules (an approximate $20 \mathrm{mM}$ concentration) in the outer LPS leaflet, where PQS is experimentally known to interact. $^{57}$ Both systems were simulated without bias and found to be stable over timescales of 200 ns. Both were then subjected to $400 \mathrm{~ns}$ of well-tempered metadynamics. Curvatures were computed on LPS head beads and the equilibrated structure of the pristine LPS/POPE patch was used as reference in both cases. To favor convergence and limit the study to small deformations, a harmonic barrier at $\Gamma=1.1$ was imposed using a harmonic wall potential.

Figure 6 shows the bending free energy profiles obtained (see Supporting Information figure S8 for their convergence). By definition, these are defined up to an additive constant: the vertical positioning of the two plots with respect to one another is thus arbitrary. However, it is clear that the energy penalty incurred upon significant deformation of the membrane patch $(\Gamma>0.6)$ is much reduced in the presence of PQS. PQS appears to limit the conformational volume of flat conformations by narrowing the corresponding free energy basin $(\Gamma \sim 0.20-0.55$ in the absence of PQS $)$ on both sides. This makes sense from a purely sterical 
Figure 5: The PQS molecule and its MARTINI representation. Grains are represented by ellipses delimiting their constitutive heavy atoms, labeled by their MARTINI types and colored by their nature (gray: apolar; green: nonpolar; red: polar).

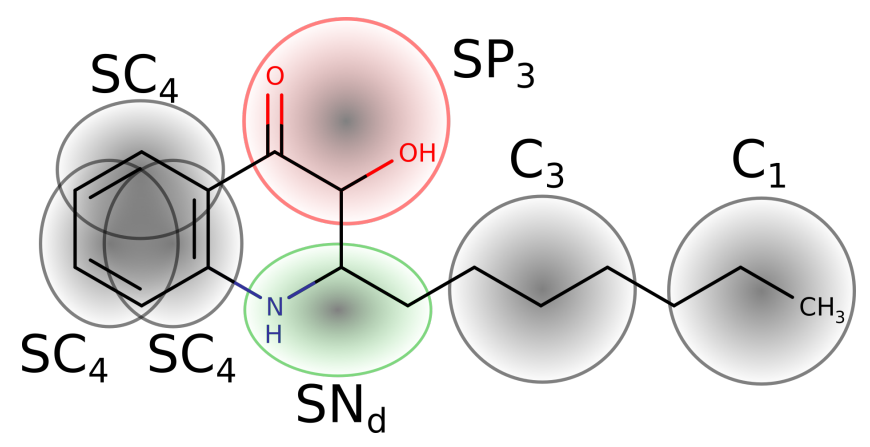

point of view: the additional space taken up by multiple, rather bulky PQS molecules forming strong interactions with the polar core of the LPS leaflet induces interleaflet tension, which is more easily relieved by bending the membrane out of plane than by compressing the membrane in-plane. It also comforts the currently adopted view of PQS-induced membrane curvature phenomena. ${ }^{57}$

However, the metadynamics simulations provide valuable additional mechanistic information. The distribution of atomic curvatures at LPS headgroups in the vicinity of PQS molecules ( $<5 \AA$ along the membrane plane) reveals that, in high- $\Gamma$ structures, $P Q S$ is more likely to be found in locally flat areas than in locally bent ones (figure 6, center panel), while in low- $\Gamma$ structures no such effect is apparent. A similar picture emerges from the analysis of area per lipid (APL) and membrane width distributions (see Supporting Information figures S9-S10). At comparable $\Gamma$ values, a wider range of $\mathrm{APL}$ values is observed in the presence of PQS than in its absence; however, on average, $\mathrm{PQS}$ molecules tend to avoid regions of very high or low APL. Bent regions also tend to feature reduced values of bilayer width; the measure of leaflet overlap along the membrane normal axis reveals that this is mainly due to the interpenetration of the lipid tails of both leaflets. PQS molecules tend to move away from such areas, possibly to limit unfavorable interactions made by the PQS polar core. From these findings emerges an overall picture in which rather mobile PQS molecules tend to congregate in the flat regions, acting as 'pincers' to bend the membrane patches inbetween. The mobility of PQS molecules within the membrane, observed in the simulations, is compatible with the recently reported lack of durable hydrogen bonding interactions between PQS and LPS. ${ }^{58}$

To the best of the author's knowledge, the evaluation of the free energetic effect of PQS on LPS membrane bending and the report of PQS distribution within a bent patch are two important novel results in a field for which knowledge at the atomic level remains very scarce.

\subsection{Limitations}

The use-cases described in the previous section demonstrate the method's potential for the accelerated simulation of membrane bending events and the quantitative study of their free energy landscapes. Nonetheless, these capabilities could be further refined by addressing some of the method's main limitations, summarized below.

The most evident limitation is the necessity to obtain a single scalar conformational coordinate from a scalar field of atomic curvatures. The solution employed here, akin to the RMSD routinely applied to the comparison of macromolecular structures, suffers from the same limitation as the former: the volume of conformational space corresponding to an isovalue of the coordinate 'blows up' at large isovalues. This problem can be partially worked around (for instance by combining several coordinates), and the low computational cost of the approach remains an advantage when it is applied to millions of MD timesteps. Nevertheless, using more robust methods leveraging Morse theory and persistence diagrams ${ }^{20}$ would help to lift the conformational degeneracy.

Other limitations stem from the ICP registration of successive reference conformations. The first one is the treatment of periodic boundary conditions. Prior to the registration process, 
Figure 6: Left: free energy profile and confidence interval for the deformation of an LPS/POPE bilayer along $\Gamma$, with or without PQS; the minimum $y$-value of each curve was arbitrary positioned at 0. Center: comparison of the distributions of atomic curvatures at LPS headgroups close to PQS molecules in the $x y$ plane (red) or randomly chosen (blue), for low and high values of $\Gamma$. Right: typical placement of PQS (yellow surfaces) inside the bilayer at $\Gamma \sim 0.8$ (green: polar LPS core; pink: lipid A; cyan: POPE leaflet; purple: $\mathrm{Ca}^{2+}$ ions; glassy surface: water).

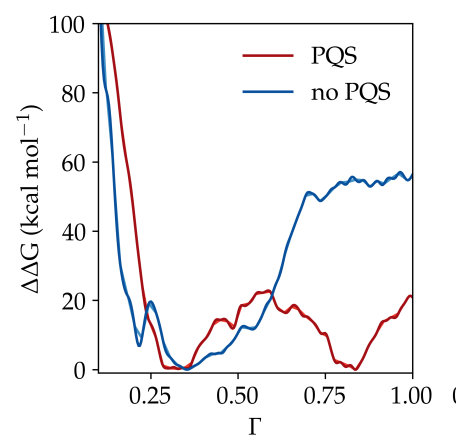

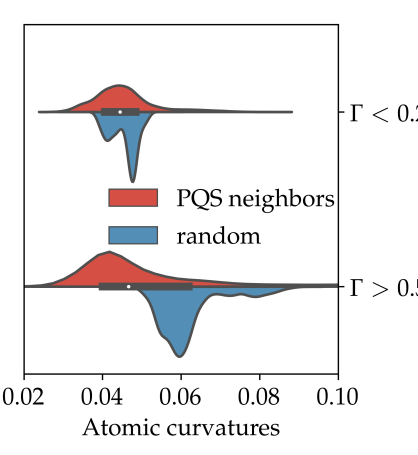

S

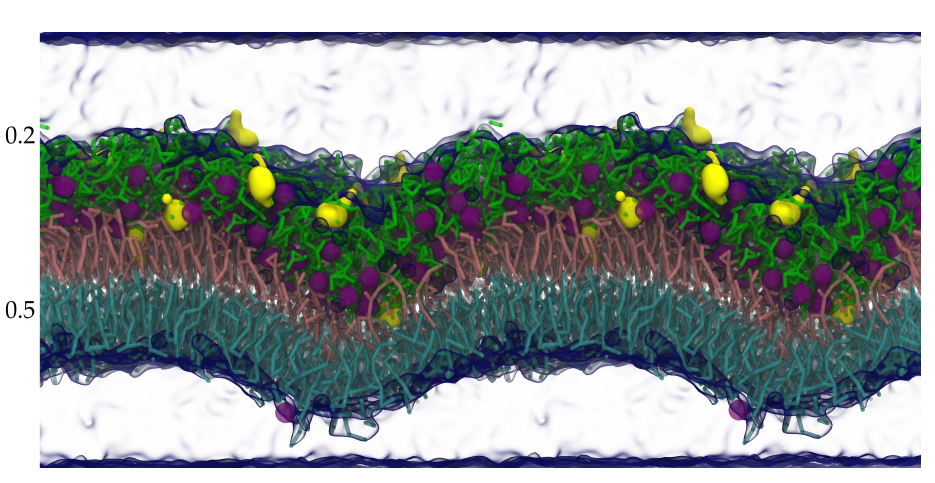

atoms in both conformations need to be placed back into the unit cell; however, a large number of atoms 'jumping' in the same direction across the cell boundary from one reference to the next could introduce a shift of surface features within the cell and deteriorate the mapping, unless the cell limits are correspondingly repositioned. In practice, such an issue was never seen to occur if the registration process is done sufficiently frequently, rendering the costly process of finding the best possible unit cell for both conformations superfluous.

A related issue originates from the mapping of atoms between conformations following the registration operation. As previously stated, atoms at time $t+\delta t$ are mapped to those at time $t$, progressively generating a chain of mappings back to the initial reference structure. The problem is that the mapping, based on a nearest-neighbor relationship, is usually not bijective: several $t+\delta t$ atoms can have the same $t$ atom as their nearest neighbor and thus become simultaneously mapped to it. Mapping two atoms at time $t+\delta t$ to the same atom at time $t$ leaves one unmapped atom at $t$; this breaks the connection to one of the reference atoms at $t+\delta t$ and all posterior time frames. As the simulation progresses, the number of such lost connections keeps rising and the number of unique reference atoms still mapped to atoms of the current conformation keeps decreasing.
The overall effect is a homogeneization of the reference curvatures, which is not a problem if these curvatures were initially homogeneous (as occurs in a flat or quasi-spherical patches) but raises issues for reference structures with localized features.

The most straightforward workaround (i) is to impose a bijective mapping by marking as unavailable atoms in the reference structure that have already been mapped and selecting the closest neighbor still available; however, as the mapping process progresses and fewer reference atoms become available, this can result in atoms that are quite far away to become associated. In addition, the result depends on the order in which the atoms are mapped: in particular, if the mapping process is performed by iterating over atom indices, and atoms with similar indices tend to be located in the same region of the surface, this can result in the quality of the mapping to be much better in some parts of the surface (those with small atom indices, that are mapped first) than others (those with large atom indices, mapped last). Possible solutions include (ii) trying several randomized mapping orders and retaining the best (which minimizes the sum of distances between mapped atoms); (iii) choosing the mapping order such that the $i+1^{t h}$ mapped atom is the furthest from the $i^{t h}$, to prevent situations in which parts of the surface are better mapped than others; (iv) find- 
ing the best mapping among all possible combinations - a very costly operation even using the polynomial-complexity Kuhn-Munkres algorithm $^{59}\left(\mathcal{O}\left(N^{4}\right)\right)$. Compared to (iv), approaches (i) to (iii) tend to give comparable results if the mapping is performed at sufficiently regular intervals, so approach (i) is used by default; the other approaches are implemented an can be selected by the user for specific scenarios. It should be kept in mind that similar issues also plague comparable approaches in spite of higher computational costs - for instance, the permutation reduction approach of Bubnis et $\mathrm{al}^{43}$ based on the minimization of a distancebased cost function is not guaranteed to converge to the global minimum. Applying the Kuhn-Munkres algorithm to a pre-filtered set of mappings (based for instance on topological persistence) could be a worthwhile refinement to the method. The filtering could also be based on the chemical nature of the mapped atoms, ${ }^{60}$ tackling the problem of multicomponent membranes for which mapping atoms based on position only can result in atoms of different chemical natures to become associated; a penalty could be applied to such mappings.

Finally, the method remains computationally costly despite its performance-oriented refinements (KD-tree, Cardano matrix diagonalization...): slowdowns by a factor of 2 to 10 compared to unbiased simulations of similar systems have been observed. Introducing explicit OpenMP/MPI parallelization into the code would surely make an important difference.

\section{Concluding remarks}

Given the ubiquitousness of membrane remodeling processes and the growing popularity of research in this field, it is the author's hope that the current work's promising results will trigger additional interest for the design of relevant collective coordinates that strive for the best tradeoff between precision, generality and performance.

Acknowledgement The calculations presented herein were performed using HPC re- sources from the MatriCS computing platform of Université de Picardie - Jules Verne, Amiens, France.

\section{Supporting Information Avail- able}

Detailed expression of covariance matrix eigenvalues and eigenvectors; additional simulation parameters; convergence of free energy profiles (FEP); areas per lipid (APL) and deuterium order parameters for POPE and DPPC patches; side view of a bent POPE bilayer; representative structures of a POPE patch at high $\Gamma$ values; reference conformation and atoms for the cylindrical POPE patch; evolution of $\Gamma$ during the formation of a POPE liposome; LPS FEP convergence; APL, leaflet overlap and PQS positions in bent LPS patches; MARTINI topology for PQS.

\section{References}

(1) Lou, H. Y.; Zhao, W.; Zeng, Y.; Cui, B. The role of membrane curvature in nanoscale topography-induced intracellular signaling. Acc. Chem. Res. 2018, 51, 1046-1053.

(2) Schmick, M.; Bastiaens, P. I. H. The interdependence of membrane shape and cellular signal processing. Cell 2014, 156, 1132-1138.

(3) Schwechheimer, C.; Kuehn, M. J. Outermembrane vesicles from Gram-negative bacteria: biogenesis and functions. Nat. Rev. Microbiol. 2015, 13, 605-619.

(4) Lind, T. K.; Darré, L.; Domene, C.; Urbanczyk-Lipkowska, Z.; Cárdenas, M.; Wacklin, H. P. Antimicrobial peptide dendrimer interacts with phosphocholine membranes in a fluidity dependent manner: A neutron reflection study combined with molecular dynamics simulations. Biochim. Biophys. Acta - Biomembr. 2015, 1848, 2075-2084. 
(5) Santi-Rocca, J.; Blanchard, N. Membrane trafficking and remodeling at the hostparasite interface. Curr. Opin. Microbiol. 2017, 40, 145-151.

(6) Hanna, M. G.; Mela, I.; Wang, L.; Henderson, R. M.; Chapman, E. R.; Edwardson, J. M.; Audhya, A. Sar1 GTPase activity is regulated by membrane curvature. $J$. Biol. Chem. 2016, 291, 1014-1027.

(7) Barbot, M.; Meinecke, M. Reconstitutions of mitochondrial inner membrane remodeling. J. Struct. Biol. 2016, 196, 20-28.

(8) Huang, C.; Quinn, D.; Sadovsky, Y.; Suresh, S.; Hsia, K. J. Formation and size distribution of self-assembled vesicles. Proc. Natl. Acad. Sci. 2017, 114, 29102915.

(9) Salzer, U.; Kostan, J.; DjinovićCarugo, K. Deciphering the BAR code of membrane modulators. Cell. Mol. Life Sci. 2017, 74, 2413-2438.

(10) Shi, Z.; Baumgart, T. Membrane tension and peripheral protein density mediate membrane shape transitions. Nat. Commun. 2015, 6, 1-8.

(11) Simunovic, M.; Bassereau, P.; Voth, G. A. Organizing membrane-curving proteins: the emerging dynamical picture. Curr. Opin. Struct. Biol. 2018, 51, 99-105.

(12) Chavent, M.; Duncan, A. L.; Sansom, M. S. Molecular dynamics simulations of membrane proteins and their interactions: From nanoscale to mesoscale. Curr. Opin. Struct. Biol. 2016, 40, 8-16.

(13) Mori, T.; Miyashita, N.; Im, W.; Feig, M.; Sugita, Y. Molecular dynamics simulations of biological membranes and membrane proteins using enhanced conformational sampling algorithms. Biochim. Biophys. Acta - Biomembr. 2016, 1858, 1635-1651.

(14) Dickson, B. M. Survey of adaptive biasing potentials: comparisons and outlook. Curr. Opin. Struct. Biol. 2017, 43, 63-67.
(15) W. Helfrich, Elastic properties of lipid bilayers - theory and possible experiments. Zeitschrift fur Naturforsch. Tl. C Biochem. Biophys. Biol. Virol. 1973, 28, 693-703.

(16) Smirnova, Y. G.; Müller, M. Calculation of membrane bending rigidity using fieldtheoretic umbrella sampling. J. Chem. Phys. 2015, 143, 243155.

(17) Hu, M.; Briguglio, J. J.; Deserno, M. Determining the Gaussian curvature modulus of lipid membranes in simulations. Biophys. J. 2012, 102, 1403-1410.

(18) Yesylevskyy, S. O.; Ramseyer, C. Determination of mean and Gaussian curvatures of highly curved asymmetric lipid bilayers: the case study of the influence of cholesterol on the membrane shape. Phys. Chem. Chem. Phys. 2014, 16, 1705217061.

(19) Gunther, D.; Jacobson, A.; Reininghaus, J.; Seidel, H.-P.; SorkineHornung, O.; Weinkauf, T. Fast and memory-efficienty topological denoising of 2D and 3D scalar fields. IEEE Trans. Vis. Comp. Graph. 2014, 20, 2585-2594.

(20) Chazal, F.; Guibas, L.; Oudot, S.; Skraba, P. Analysis of scalar fields over point cloud data. Proc. Twent. Annu. ACM-SIAM Symp. Discret. Algorithms 2009, 1021-1030.

(21) Gyulassy, A.; Natarajan, V.; Pascucci, V.; Bremer, P. T.; Hamann, B. A topological approach to simplification of threedimensional scalar functions. IEEE Trans. Vis. Comput. Graph. 2006, 12, 474-484.

(22) Masone, D.; Uhart, M.; Bustos, D. M. Bending lipid bilayers: a closed-form collective variable for effective free-energy landscapes in quantitative biology. $J$. Chem. Theory Comput. 2018, 14, 22402245 . 
(23) Hoppe, H.; DeRose, T.; Duchamp, T.; McDonald, J.; Stuetzle, W. Surface reconstruction from unorganized points. Proc. 19th Annu. Conf. Comput. Graph. Interact. Tech. SIGGRAPH '92. New York, NY, USA, 1992; pp 71-78.

(24) Buchoux, S. Structural bioinformatics FATSLiM : a fast and robust software to analyze MD simulations of membranes. Bioinformatics 2017, 33, 133-134.

(25) Co, C. S.; Heckel, B.; Hagen, H.; Hamann, B.; Joy, K. I. Hierarchical clustering for unstructured volumetric scalar fields. Proc. IEEE Vis. Conf. 2003; pp 325-332.

(26) Rabbani, T.; van den Heuvel, F. A.; Vosselman, G. Segmentation of point clouds using smoothness constraint. Int. Arch. Photogram. Remote Sens. 2006, 36, 248253.

(27) Tierny, J.; Pascucci, V. Generalized topological simplification of scalar fields on surfaces. IEEE Trans. Vis. Comput. Graph. 2012, 18, 2005-2013.

(28) Thomas, D. M.; Natarajan, V. Multiscale symmetry detection in scalar fields by clustering contours. IEEE Trans. Vis. Comput. Graph. 2014, 20, 2427-2436.

(29) Lee, J.; Kim, S.; Kim, S. J. Mesh segmentation based on curvatures using the GPU. Multimed. Tools Appl. 2014, 74, 34013412 .

(30) Cardano, G. Ars magna or The Rules of Algebra; Dover Press, 1545.

(31) Kopp, J. Efficient numerical diagonalization of hermitian $3 \times 3$ matrices. Int. $J$. Mod. Phys. C 2008, 19, 523-548.

(32) Hascoet, L.; Pascual, V. The Tapenade automatic differentiation tool: principles, model, and specification. ACM Trans. Math. Soft. 2013, 39, 20.
(33) Metzler, R.; Jeon, J. H.; Cherstvy, A. G. Non-Brownian diffusion in lipid membranes: Experiments and simulations. Biochim. Biophys. Acta - Biomembr. 2016, 1858, 2451-2467.

(34) Bouvier, B.; Zakrzewska, K.; Lavery, R. Protein-DNA recognition triggered by a DNA conformational switch. Angew. Chem. 2011, 50, 6516-6518.

(35) Basciu, A.; Malloci, G.; Pietrucci, F.; Bonvin, A. M.; Vargiu, A. V. Holo-like and druggable protein conformations from enhanced sampling of binding pocket volume and shape. J. Chem. Inf. Model. 2019, 59, 1515-1528.

(36) Spiwok, V.; Králová, B. Metadynamics in the conformational space nonlinearly dimensionally reduced by Isomap. J. Chem. Phys. 2011, 135, 224504.

(37) Camilloni, C.; Pietrucci, F. Advanced simulation techniques for the thermodynamic and kinetic characterization of biological systems. Adv. Phys. X 2018, 3, 885-916.

(38) Blanco, J. L.; Rai, P. K. Nanoflann: a $\mathrm{C}++11$ header-only library for Nearest Neighbor (NN) with KD-trees. 2014; https://github.com/jlblancoc/ nanoflann.

(39) Tribello, G. A.; Bonomi, M.; Branduardi, D.; Camilloni, C.; Bussi, G. PLUMED 2: New feathers for an old bird. Comput. Phys. Comm. 2014, 185, 604613.

(40) Abraham, M. J.; Murtola, T.; Schulz, R.; Páll, S.; Smith, J. C.; Hess, B.; Lindahl, E. GROMACS: High performance molecular simulations through multi-level parallelism from laptops to supercomputers. SoftwareX 2015, 1, 19-25.

(41) Arnarez, C.; Uusitalo, J. J.; Masman, M. F.; Ingólfsson, H. I.; de Jong, D. H.; Melo, M. N.; Periole, X.; de Vries, A. H.; Marrink, S. J. Dry Martini, a coarse-grained force field 
for lipid membrane simulations with implicit solvent. J. Chem. Theory Comput. 2015, 11, 260-275.

(42) Kollmitzer, B.; Heftberger, P.; Rappolt, M.; Pabst, G. Monolayer spontaneous curvature of raft-forming membrane lipids. Soft Matter 2013, 9, 10877-10884.

(43) Bubnis, G.; Risselada, H. J.; Grubmüller, H. Exploiting lipid permutation symmetry to compute membrane remodeling free energies. Phys. Rev. Lett. 2016, $117,1-6$.

(44) Qi, Y.; Cheng, X.; Han, W.; Jo, S.; Roux, B.; Schulten, K.; Im, W. CHARMM-GUI PACE CG Builder for solution, micelle, bilayer and vesicle simulations. J. Chem. Inf. Model. 2014, 54, 1003-1009.

(45) Hudiyanti, D.; Radifar, M.; Raharjo, T. J.; Narsito, N.; Noegrohati, S. A coarsegrained molecular dynamics simulation using NAMD package to reveal aggregation profile of phospholipids self-assembly in water. J. Chem. 2014, 2014.

(46) Shinoda, W.; DeVane, R.; Klein, M. L. Computer simulation studies of selfassembling macromolecules. Curr. Opin. Struct. Biol. 2012, 22, 175-186.

(47) Whiteley, M.; Diggle, S. P.; Greenberg, E. P. Progress in and promise of bacterial quorum sensing research. Nature 2017, 551, 313-320.

(48) Tacconelli, E.; Magrini, N. Global priority list of antibiotic-resistant bacteria to guide research, discovery, and development of new antibiotics. 2017; https://www . who.int/medicines/publications/ global-priority-list-antibiotic-resistanten/.

(49) Mashburn, L. M.; Whiteley, M. Membrane vesicles traffic signals and facilitate group activities in a prokaryote. Nature 2005, $437,422-425$.
(50) Veith, P. D.; Chen, Y.-Y.; Gorasia, D. G.; Chen, D.; Glew, M. D.; O'Brien-Simpson, N. M.; Cecil, J. D.; Holden, J. A.; Reynolds, E. C. Porphyromonas gingivalis outer membrane vesicles exclusively contain outer membrane and periplasmic proteins and carry a cargo enriched with virulence factors. J. Proteome Res. 2014, 13, 2420-2432.

(51) Lappann, M.; Otto, A.; Becher, D.; Vogel, U. Comparative proteome analysis of spontaneous outer membrane vesicles and purified outer membranes of Neisseria meningitidis. J. Bacteriol. 2013, 195, 4425-4435.

(52) Wang, S.; Gao, J.; Wang, Z. Outer membrane vesicles for vaccination and targeted drug delivery. Wiley Interdiscip. Rev. Nanomed. Nanobiotechnol. 2019, 11, $1-16$.

(53) Khalid, S.; Piggot, T. J.; Samsudin, F. Atomistic and Coarse Grain Simulations of the Cell Envelope of Gram-Negative Bacteria: What Have We Learned? Acc. Chem. Res. 2019, 52, 180-188.

(54) De Jong, D. H.; Singh, G.; Bennett, W. F.; Arnarez, C.; Wassenaar, T. A.; Schäfer, L. V.; Periole, X.; Tieleman, D. P.; Marrink, S. J. Improved parameters for the martini coarse-grained protein force field. J. Chem. Theory Comput. 2013, 9, 687-697.

(55) Oosten, B. V.; Harroun, T. A. A MARTINI extension for Pseudomonas aeruginosa PAO1 lipopolysaccharide. J. Mol. Graph. Model. 2016, 63, 125-133.

(56) Kirschner, K. N.; Lins, R. D.; Maass, A.; Soares, T. A. A glycam-based force

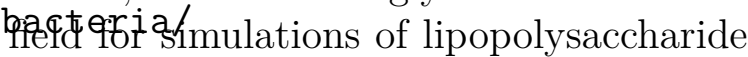
membranes: parametrization and validation. J. Chem. Theory Comput. 2012, 8, 4719-4731.

(57) Schertzer, J. W.; Whiteley, M. A bilayercouple model of bacterial outer membrane 
vesicle biogenesis. MBio 2012, 3, e00297-

11.

(58) Li, A.; Schertzer, J. W.; Yong, X. Molecular conformation affects the interaction of the Pseudomonas quinolone signal with the bacterial outer membrane. J. Biol. Chem. 2019, 294, 1089-1094.

(59) Munkres, J. Algorithms for the assignment and transportation problems. J. Soc. Indust. Appl. Math. 1957, 5, 32-38.

(60) Temelso, B.; Mabey, J. M.; Kubota, T.; Appiah-Padi, N.; Shields, G. C. ArbAlign: A tool for optimal alignment of arbitrarily ordered isomers using the Kuhn-Munkres algorithm. J. Chem. Inf. Model. 2017, 57, $1045-1054$. 


\section{Graphical TOC Entry}

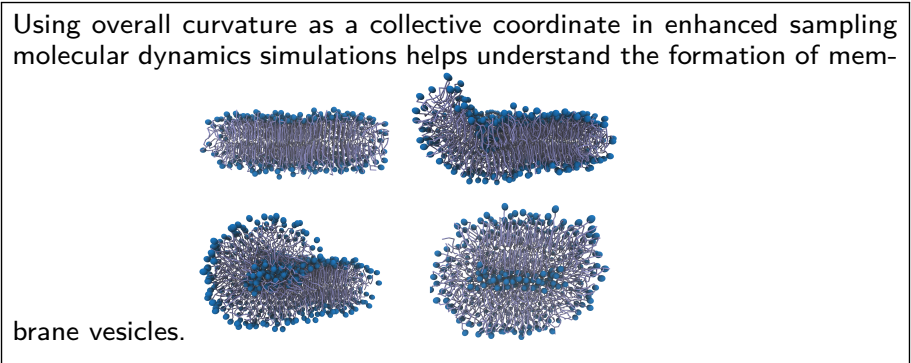

